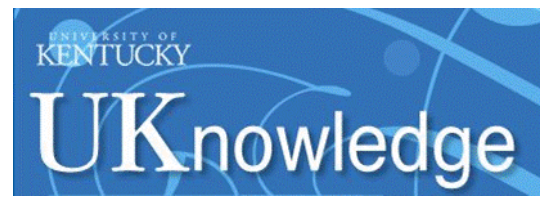

University of Kentucky

UKnowledge

4-1-2015

\title{
General Method to Predict Voltage-Dependent Ionic Conduction in a Solid Electrolyte Coating on Electrodes
}

Jie Pan

University of Kentucky, jie.pan@uky.edu

Yang-Tse Cheng

University of Kentucky, yang.t.cheng@uky.edu

Yue Qi

Michigan State University

Follow this and additional works at: https://uknowledge.uky.edu/cme_facpub

Part of the Chemical Engineering Commons, and the Materials Science and Engineering Commons Right click to open a feedback form in a new tab to let us know how this document benefits you.

\section{Repository Citation}

Pan, Jie; Cheng, Yang-Tse; and Qi, Yue, "General Method to Predict Voltage-Dependent lonic Conduction in a Solid Electrolyte Coating on Electrodes" (2015). Chemical and Materials Engineering Faculty Publications. 12.

https://uknowledge.uky.edu/cme_facpub/12

This Article is brought to you for free and open access by the Chemical and Materials Engineering at UKnowledge. It has been accepted for inclusion in Chemical and Materials Engineering Faculty Publications by an authorized administrator of UKnowledge. For more information, please contact UKnowledge@lsv.uky.edu. 


\title{
General Method to Predict Voltage-Dependent Ionic Conduction in a Solid Electrolyte Coating on Electrodes
}

\author{
Digital Object Identifier (DOI) \\ http://dx.doi.org/10.1103/PhysRevB.91.134116 \\ Notes/Citation Information \\ Published in Physical Review B: Condensed Matter and Materials Physics, v. 91, no. 13, article 134116, p. \\ $1-9$. \\ (C2015 American Physical Society
}

The copyright holder has granted permission for posting the article here. 


\title{
General method to predict voltage-dependent ionic conduction in a solid electrolyte coating on electrodes
}

\author{
Jie Pan* and Yang-Tse Cheng ${ }^{\dagger}$ \\ Department of Chemical and Materials Engineering, University of Kentucky, Lexington, Kentucky 40506-0046, USA
}

\author{
Yue $\mathrm{Qi}^{\ddagger}$ \\ Department of Chemical Engineering and Materials Science, Michigan State University, East Lansing, Michigan 48824-1226, USA
}

(Received 2 December 2014; revised manuscript received 3 April 2015; published 30 April 2015)

\begin{abstract}
Understanding the ionic conduction in solid electrolytes in contact with electrodes is vitally important to many applications, such as lithium ion batteries. The problem is complex because both the internal properties of the materials (e.g., electronic structure) and the characteristics of the externally contacting phases (e.g., voltage of the electrode) affect defect formation and transport. In this paper, we developed a method based on density functional theory to study the physics of defects in a solid electrolyte in equilibrium with an external environment. This method was then applied to predict the ionic conduction in lithium fluoride (LiF), in contact with different electrodes which serve as reservoirs with adjustable Li chemical potential $\left(\mu_{\mathrm{Li}}\right)$ for defect formation. $\mathrm{LiF}$ was chosen because it is a major component in the solid electrolyte interphase (SEI) formed on lithium ion battery electrodes. Seventeen possible native defects with their relevant charge states in $\mathrm{LiF}$ were investigated to determine the dominant defect types on various electrodes. The diffusion barrier of dominant defects was calculated by the climbed nudged elastic band method. The ionic conductivity was then obtained from the concentration and mobility of defects using the Nernst-Einstein relationship. Three regions for defect formation were identified as a function of $\mu_{\mathrm{Li}}$ : (1) intrinsic, (2) transitional, and (3) p-type region. In the intrinsic region (high $\mu_{\mathrm{Li}}$, typical for $\mathrm{LiF}$ on the negative electrode), the main defects are Schottky pairs and in the $p$-type region (low $\mu_{\mathrm{Li}}$, typical for $\mathrm{LiF}$ on the positive electrode) are $\mathrm{Li}$ ion vacancies. The ionic conductivity is calculated to be approximately $10^{-31} \mathrm{~S} \mathrm{~cm}^{-1}$ when $\mathrm{LiF}$ is in contact with a negative electrode but it can increase to $10^{-12} \mathrm{~S} \mathrm{~cm}^{-1}$ on a positive electrode. This insight suggests that divalent cation (e.g., $\mathrm{Mg}^{2+}$ ) doping is necessary to improve $\mathrm{Li}$ ion transport through the engineered $\mathrm{LiF}$ coating, especially for LiF on negative electrodes. Our results provide an understanding of the influence of the environment on defect formation and demonstrate a linkage between defect concentration in a solid electrolyte and the voltage of the electrode.
\end{abstract}

DOI: 10.1103/PhysRevB.91.134116

PACS number(s): 66.30.Dn, 66.30.Lw, 82.47.Aa, 71.15.Mb

\section{INTRODUCTION}

The origin of ionic conduction in solid electrolytes is defect formation and transport in ionic materials [1-4]. At a defined temperature, the internal equilibria of defects depend on two aspects: (1) the atomic structure of the defects and (2) the electronic band structure of the material $[3,5,6]$. However, in reality, an ionic material of interest always operates in an environment (e.g., $\mathrm{PbO}$ in oxygen gas [3]; Li ionic conductors coated on electrodes). In this situation, due to the formation of defects, the compound can deviate from the exact stoichiometric composition. As a result, the concentration of defects varies with the environment in which the ionic material is in a thermodynamic equilibrium [3]. For example, by increasing the partial oxygen pressure $P_{\mathrm{O}_{2}}$ (equivalently chemical potential of oxygen $\mu_{O_{2}}=\mu_{O_{2}}^{\circ}+R T \ln \frac{P_{O_{2}}}{P^{\circ}}$ [3]) passing over $\mathrm{PbO}$, the concentration of oxygen vacancies decreases while that of the oxygen interstitials increases as depicted in the Kröger-Vink or Brouwer diagram [3,5]. Therefore, the environment can be treated as a "tuner" that can change the concentrations of defects in a material. In general, the environment is not only limited to the gas phase, but can be liquid or solid phase,

\footnotetext{
*jie.pan@uky.edu

†yang.t.cheng@uky.edu

†yueqi@egr.msu.edu
}

such as electrodes on which the solid electrolyte is coated. Similarly, the concentrations of defects in the solid electrolyte would be electrode voltage dependent according to the Nernst equation [ $\left.V=-\frac{1}{z_{i} F}\left(\mu_{M}^{\text {cathode }}-\mu_{M}^{\text {anode }}\right)\right]$ [7]. The goal of this work is to develop a method to study defect physics and, thus, ionic conduction in a material in contact with an external environment, e.g., a solid electrolyte on various electrodes for lithium ion batteries (LIBs).

Recently, solid electrolytes attracted a lot of research emphasizes not only because the promising future of all-solid batteries [4,8-16], but also due to their importance as an interfacial layer between electrodes and liquid electrolytes, known as a solid electrolyte interphase (SEI) [17,18]. The performance of liquid electrolyte based LIBs relies on forming a stable SEI on the electrode surface $[17,18]$. In theory, an ideal SEI is expected to be ionic conducting [19-21], electrical insulating [22-24], and mechanically stable [25,26]. However, the characters of a natural SEI depend on many factors (e.g., property of electrode and electrolyte molecules) and a stable passivating layer is not always formed. For example, silicon (Si) electrode, one of the most promising negative electrodes with the highest theoretical capacity [27], cannot maintain a stable interphase [26] due to its large volume expansion [28-31]. This leads to a continuous capacity loss and degradation of the battery. Recently, surface coatings [32-35] and electrolyte additives [36] have been proposed as two promising ways to modify SEIs. In the first approach, surface 
coatings, such as $\mathrm{Al}_{2} \mathrm{O}_{3}, \mathrm{TiO}_{2}$, and $\mathrm{AlF}_{3}$, have been designed to serve as an artificial SEI to mitigate electrochemical and mechanical degradation of the electrodes [32-35,37,38]. It was believed that solid electrolyte thin film coatings, also known as engineered artificial SEIs, were more stable than naturally formed SEIs in maintaining passivation, preventing continuous liquid electrolyte molecule decomposition, and protecting the electrodes from further degradation [32-35]. The second approach, by adding small amounts of electrolyte additives (e.g., vinylene carbonate [39,40], fluoroethylene carbonate [41-47]), the performance of the electrodes was improved with a longer cycling life. The additives have been shown to change the inorganic components in the SEI (e.g., increased formation of lithium fluoride) [41-47]. The mechanisms responsible for the two promising approaches are not well understood. A theoretical approach is therefore needed to understand the mechanism of ionic and electronic conduction in SEI components.

A theoretical method to understand ionic conduction in a solid electrolyte should include two parts: (1) the concentration of defects in the solid electrolyte in contact with electrodes and (2) the transport of the dominant defects (e.g., Li vacancy). The dependence of charged defect reactions on the chemical potential of electrons (i.e., Fermi energy) has been observed in various material systems [48-51]. More generally, Zhang and Northrup [52] developed a first principles based method and demonstrated that defect concentration is a function of the chemical potential of atomic constituents. This formalism has been successfully applied to study multiple defect physics, e.g., defect compensation in $\mathrm{ZnSe} \mathrm{[53],} \mathrm{zinc} \mathrm{vacancies} \mathrm{as}$ the dominant intrinsic acceptor defects in $\mathrm{ZnO}$ [54], and nitrogen vacancies as the dominant intrinsic donor defects in p-type GaN [55]. In 2004, Van de Walle and Neugebauer [56] published a comprehensive review article on this method and its applications to study defect physics in III-nitrides.

However, to benefit battery design, it is necessary to link defect reactions to an electrochemically measurable parameter, such as the open circuit voltage of the electrode. In this work, we extended this method [52-56] based on density functional theory to calculate the ionic conductivity in a solid electrolyte on electrodes. In our approach, electrodes are modeled as lithium $(\mathrm{Li})$ reservoirs with a range of $\mathrm{Li}$ chemical potential values $\left(\mu_{\mathrm{Li}}\right)$ and $\mu_{\mathrm{Li}}$ can be related to the voltage of electrodes. Through this process, the Fermi energy dependent defect reactions in a solid electrolyte can be related to the voltage of neighboring electrodes. Shi et al. have taken a similar approach to investigate $\mathrm{Li}$ point defects in $\mathrm{Li}_{2} \mathrm{CO}_{3}[20,21]$ as a function of the open-circuit voltage of electrode that $\mathrm{Li}_{2} \mathrm{CO}_{3}$ is coated on. In their work, $\mathrm{Li}$ interstitials, $\mathrm{Li}$ vacancies, and Li Frenkel pairs were considered as possible point defects, since carbon and oxygen were covalently bonded forming the oxocarbon anion $\mathrm{CO}_{3}^{2-}$. In general, both cation and anion defects need to be considered in ionic materials, e.g., lithium fluoride (LiF). In addition, there remain other technical challenges in their method, such as the determination of the effective density of states at a finite temperature from density functional approximations and estimation of contribution of paired defects to ionic conduction.

In this study, our interest focuses on ionic conduction in $\mathrm{LiF}$ coated on various electrodes with rigorous considerations of the remaining technical challenges. $\mathrm{LiF}$ is one of the promising engineered SEI coating materials on the electrodes (e.g., Si) of LIBs, since the improved performance of electrodes has been linked to the increased concentration of $\mathrm{LiF}$ in the natural SEI [41-47,57]. In addition, perfect LiF crystals have a wide band gap [58-60], and can thus block the electron leakage to the electrolyte from the electrodes. In the present work, as a development of a general approach to evaluate all possible point defects, we studied ionic conductivity of a total of seventeen possible native defects with their relevant charge states in $\mathrm{LiF}$ on the surface of different electrodes. In addition, using this first principles based ionic defect calculation method, we demonstrate the dependence of defect formation and, accordingly, the ionic conduction as a function of the chemical potential of Li in the electrodes. Finally, the possibility of $\mathrm{LiF}$ as an engineered artificial SEI is discussed from the perspective of ionic and electrical conduction.

\section{METHODOLOGY}

\section{A. Thermodynamics of defects}

In ionic crystals, typical types of defects include interstitials, vacancies, Frenkel pairs, and Schottky pairs [1-3]. Table I summarizes possible defects with various charge states in $\mathrm{LiF}$ and their notations used in this paper. In our model, defects are equilibrated with external Li reservoirs (known as external defect equilibria [3], in which deviation from the exact stoichiometric composition is allowed). As a result, the formation energy of a defect $i$ with charge $q$ is defined

TABLE I. Summary of defect types in LiF.

\begin{tabular}{lcl}
\hline \hline $\begin{array}{l}\text { Notation } \\
(i)\end{array}$ & $\begin{array}{c}\text { Kröger-Vink } \\
\text { notation }[3,5,6]\end{array}$ & \multicolumn{1}{c}{ Defect types } \\
\hline $\mathrm{Li}_{i}$ & $\mathrm{Li}_{i}^{\times}$ & Li neutral interstitial \\
$\mathrm{Li}_{i}^{+}$ & $\mathrm{Li}_{i}^{\bullet}$ & Li positively charged interstitial \\
$F_{i}$ & $F_{i}^{\times}$ & F neutral interstitial \\
$F_{i}^{-}$ & $F_{i}^{\prime}$ & Li negatively charged interstitial \\
$V_{\mathrm{Li}}$ & $V_{\mathrm{Li}}^{\times}$ & Li neutral vacancy \\
$V_{\mathrm{Li}}^{-}$ & $V_{\mathrm{Li}}^{\prime}$ & Li negatively charged vacancy \\
$V_{F}$ & $V_{F}^{\times}$ & F neutral vacancy \\
$V_{F}^{+}$ & $V_{F}^{\bullet}$ & F positively charged vacancy \\
$F P_{\mathrm{Li}}^{\mathrm{a}}$ & $\mathrm{Li}_{i}^{\circ}+V_{\mathrm{Li}}^{\prime}$ & Li neutral Frenkel pair \\
$F P_{\mathrm{Li}}^{+\mathrm{a}}$ & $\mathrm{Li}_{i}^{\bullet}+V_{\mathrm{Li}}^{\times}$ & Li positively charged Frenkel pair \\
$F P_{\mathrm{Li}}^{-\mathrm{a}}$ & $\mathrm{Li}_{i}^{\times}+V_{\mathrm{Li}}^{\prime}$ & Li negatively charged Frenkel pair \\
$F P_{F}^{\mathrm{a}}$ & $F_{i}^{\prime}+V_{F}^{\bullet}$ & F neutral Frenkel pair \\
$F P_{F}^{+\mathrm{a}}$ & $F_{i}^{\times}+V_{F}^{\bullet}$ & F positively charged Frenkel pair \\
$F P_{F}^{-\mathrm{a}}$ & $F_{i}^{\prime}+V_{F}^{\times}$ & F negatively charged Frenkel pair \\
$S P^{\mathrm{a}}$ & $V_{\mathrm{Li}}^{\prime}+V_{F}^{\bullet}$ & Neutral Schottky pair \\
$S P^{+\mathrm{a}}$ & $V_{\mathrm{Li}}^{\times}+V_{F}^{\bullet}$ & Positively charged Schottky pair \\
$S P^{-\mathrm{a}}$ & $V_{\mathrm{Li}}^{\prime}+V_{F}^{\times}$ & Negatively charged Schottky pair \\
\hline
\end{tabular}

${ }^{\text {a The formation energies of paired defects (Frenkel pair and Schottky }}$ pair) are dependent on their distances of separation. For each type of pair, the formation energies of the nearest-, second nearest-, and third nearest-neighbor pairs were calculated and the one with the lowest formation energy was included in Eq. (3). In addition, another scenario is the dilute pair (noninteracting) when two dilute point defects (e.g., $V_{\mathrm{Li}}^{-}, V_{F}^{+}$) have the same formation energy. 
as $[20,21,52-56]$

$$
\begin{aligned}
E_{f}(i, q)= & {\left[E_{\mathrm{tot}}(i, q)-n_{\mathrm{Li}} \mu_{\mathrm{Li}}-n_{F} \mu_{F}\right.} \\
& \left.+q\left(\varepsilon_{F}+E_{V}+\Delta V\right)\right]-E_{\mathrm{tot}}^{\text {bulk }},
\end{aligned}
$$

where $E_{\mathrm{tot}}(i, q)$ and $E_{\mathrm{tot}}^{\text {bulk }}$ are the calculated energies for the $\mathrm{LiF}$ supercell with and without one defect i. $E_{V}\left(E_{V}=0\right)$ is the valence band top of the bulk LiF. $\Delta V$ is the electrostatic energy correction term to align the valence band maximum of the defected cells with that in the bulk $\operatorname{LiF}[53,56] . n_{\mathrm{Li}}\left(n_{F}\right)$ is defined as the number of different $\mathrm{Li}(\mathrm{F})$ atoms compared with imperfect and perfect $\mathrm{LiF}$ supercells (e.g., for $\mathrm{Li}_{i}, n_{\mathrm{Li}}=1$ and $\left.n_{F}=0\right) . \mu_{\mathrm{Li}}$ and $\mu_{F}$ are the chemical potential of $\mathrm{Li}$ and $\mathrm{F}$ in their reservoirs. In our calculation, $\mu_{\mathrm{Li}}$ is a free parameter depending on the properties of coated electrodes; $\mu_{F}$ is approximated by assuming that $\mathrm{LiF}$ is the most stable compound for $\mathrm{F}$ to form

$$
\mu_{F}=\mu_{L i F}-\mu_{\mathrm{Li}} .
$$

In addition, it is necessary to determine the bounds on $\mu_{\mathrm{Li}}$ in Eq. (1) [56]. $\mu_{\mathrm{Li}}$ depends on the electrode on which $\mathrm{LiF}$ is coated, i.e., $\mathrm{LiF}$ coated on the anode or cathode. On the one hand, we consider that $\mathrm{LiF}$ is subjected to an upper bound when LiF is coated on Li metal: $\mu_{\mathrm{Li}}^{\max }=\mu_{\mathrm{Li}}^{\text {metal }} \approx-2.02 \mathrm{eV}$. If we push $\mu_{\mathrm{Li}}$ higher, $\mathrm{Li}$ metal would be deposited with $F^{-}$ ion dissolved in electrolyte. On the other hand, the lower bound is considered when LiF is coated on the cathode: $\mu_{\mathrm{Li}}^{\mathrm{min}}=$ $\mu_{\text {LiF }}-\mu_{F}^{F_{2}(\mathrm{gas})} \approx-9.71 \mathrm{eV}$. Under this extreme condition, $F_{2}$ gas would be generated with $\mathrm{Li}^{+}$dissolved in the electrolyte. However, for battery applications, the typical $\mu_{\mathrm{Li}}$ is higher than this limit (i.e., $\mu_{\mathrm{Li}} \approx-6.6 \mathrm{eV}$ corresponding to about 4.6 volts against $\mathrm{Li}$ metal) [61]. As a result, we use $\mu_{\mathrm{Li}} \approx-6.6 \mathrm{eV}$ as $\mu_{\mathrm{Li}}^{\mathrm{min}}$ in our study.

The Fermi energy $\left(\varepsilon_{F}\right)$, which is an unknown parameter, is referenced to the valence band top in Eq. (1). In perfect $\mathrm{LiF}$, the Fermi level lies in the middle of the band gap; however, defects can create additional states in the band gap and vary the Fermi level. In our calculation, $\varepsilon_{F}$ is determined by imposing the charge neutrality $[20,21,52]$ by balancing free electrons $\left(n_{\ominus}\right)$, free holes $\left(n_{\oplus}\right)$, and charged defects

$$
\sum_{i} q_{i} S(i, q)=n_{\ominus}-n_{\oplus},
$$

where $S(i, q)$ and $q_{i}$ are the concentration and the associated charge of defect species, $i$, respectively. For pairs (e.g., Frenkel and Schottky pairs), there are energy penalties to separate the nearest-neighbor pairs (e.g., for SP, the pair with $2.52 \AA$ farther away in distance has $\sim 0.3 \mathrm{eV}$ higher in formation energy). Therefore, only the nearest-neighbor Frenkel (Schottky) pairs were included in charge balancing. At thermodynamic equilibrium, the concentration of defect $i$ with formation energy $E_{f}(i, q)$ at a finite temperature $T$ is

$$
S(i, q)=N_{s}(i) e^{-E_{f}(i, q) / k_{B} T},
$$

where $N_{s}(i)$ is the number of sites where defect $i$ can be generated per unit volume. $n_{\ominus}\left(n_{\oplus}\right)$ are the concentration of electrons (holes) in the conduction (valence) band. At a given temperature, they are defined as

$$
\begin{aligned}
& n_{\ominus}=\int_{E_{C}}^{\infty} d \varepsilon f(\varepsilon) D(\varepsilon), \\
& n_{\oplus}=\int_{-\infty}^{E_{V}} d \varepsilon(1-f(\varepsilon)) D(\varepsilon),
\end{aligned}
$$

where $E_{C}\left(E_{V}\right)$ is the bottom (top) of the conduction (valence) band, $f(\varepsilon)=1 /\left(1+\exp \left(\frac{\varepsilon-\varepsilon_{F}}{k_{B} T}\right)\right)$ is the Fermi-Dirac distribution, and $D(\varepsilon)$ is the calculated electronic density of states. Since, for LiF, $\varepsilon-\varepsilon_{F} \gg k_{B} T$ ( $T$ is $300 \mathrm{~K}$ in our calculation), Eq. (5) can be approximated by

$$
\begin{aligned}
& n_{\ominus}=N_{C} e^{-\left(E_{C}-\varepsilon_{F}\right) / k_{B} T}, \\
& n_{\oplus}=N_{V} e^{-\left(\varepsilon_{F}-E_{V}\right) / k_{B} T},
\end{aligned}
$$

where $N_{C}\left(N_{V}\right)$ is the effective density of states in the conduction (valence) band

$$
\begin{aligned}
& N_{C}=\int_{E_{C}}^{\infty} d \varepsilon e^{-\left(\varepsilon-E_{C}\right) / k_{B} T} D(\varepsilon), \\
& N_{V}=\int_{-\infty}^{E_{V}} d \varepsilon e^{\left(\varepsilon-E_{V}\right) / k_{B} T} D(\varepsilon) .
\end{aligned}
$$

For materials with wide band gaps (e.g., alkali halides), only the energy levels near the bottom (top) of the conduction (valence) band can be occupied (unoccupied). Therefore, we approximate $N_{C}\left(N_{V}\right)$ as

$$
\begin{aligned}
& N_{C} \approx \int_{E_{C}}^{E_{C}+\Delta} d \varepsilon D(\varepsilon), \\
& N_{V} \approx \int_{E_{V}-\Delta}^{E_{V}} d \varepsilon D(\varepsilon),
\end{aligned}
$$

where $\Delta$ is a small energy interval above the conduction band (below the valence band for $N_{V}$ ).

\section{B. Defect diffusion and ionic conduction}

The ionic conductivity in ionic materials depends on both the formation and the diffusion of defects [3]. The flux density of a defect $(i, q)$ under a concentration gradient and a potential gradient can be described as the sum of Fick's and Ohm's law [3]

$$
\mathbf{j}(i, q)=-\frac{S(i, q) D(i, q)}{R T}(\nabla \ln S(i, q)+q F \nabla \phi),
$$

where $D(i, q)$ is the diffusion coefficient of the defect $(i, q), F$ is the Faraday constant, and $\phi$ is the electric potential. $D(i, q)$ can be calculated by Arrhenius equation at a finite temperature $T[62]$

$$
\begin{aligned}
D(i, q) & =g f \nu^{*}(\Delta x)^{2} \exp \left(-E_{m}(i, q) / k_{B} T\right) \\
& \approx \frac{1}{2} v^{*}(\Delta x)^{2} \exp \left(-E_{m}(i, q) / k_{B} T\right),
\end{aligned}
$$

where $v^{*}$ is the typical phonon frequency (e.g., $v^{*} \approx 10^{13}$ $\mathrm{Hz}$ along [110] direction [63]), $\Delta x$ is the net travel distance in each hop, $g$ is the geometric factor, $f\left(f=\frac{1+\sum_{j=1}^{z} Q_{j} \cos \theta_{j}}{1-\sum_{j=1}^{z} Q_{j} \cos \theta_{j}}\right.$ [64], where $Q_{j}$ is the jumping possibility along angle $\theta_{j}$ ) is the correlation factor, and $E_{m}(i, q)$ is the diffusion barrier of the defect $(i, q)$. For $1 \mathrm{D}$ diffusion approximation (i.e., $V_{\mathrm{Li}}^{-}$ 
diffuses along [11̄0] direction), $\sum_{j=1}^{z} Q_{j} \cos \theta_{j}=\frac{1}{2}[\cos (0)+$ $\cos (\pi)]=0$ and $g=\frac{1}{2}$.

By assuming that the concentration of defects is spatially uniform, the flux density depends only on the applied potential gradient

$$
\mathbf{j}(i, q)=-q F \frac{S(i, q) D(i, q)}{R T} \nabla \phi .
$$

Multiplying $q F$ to both sides of Eq. (11), the flux density $\mathbf{j}(i, q)$ is converted to the current density

$$
\mathbf{i}(i, q)=-q^{2} F^{2} \frac{S(i, q) D(i, q)}{R T} \nabla \phi .
$$

From Eq. (12), we can calculate the contribution of defect $(i, q)$ to ionic conduction by

$$
\sigma(i, q)=-\frac{\mathbf{i}(i, q)}{\nabla \phi}=q^{2} F^{2} \frac{S(i, q) D(i, q)}{R T},
$$

and the total ionic conduction is the sum of the contributions from main defects in the system

$$
\sigma=\sum_{i} \sigma(i, q)
$$

\section{Computational details}

In this study, the energies and electronic density of states were calculated by density functional theory (DFT) with plane wave basis sets in the Vienna ab initio simulation package (VASP) $[65,66]$. The exchange-correlation functional was approximated by the generalized gradient approximation (GGA) in the Perdew-Burke-Ernzerhof (PBE) flavor [67]. Projector-augmented-wave (PAW) potentials were used to mimic the ionic potentials [68]. The crystal $\mathrm{LiF}$ has the sodium chloride face-centered cubic structure (space group: $F m \overline{3} m$, No. $225[2,69])$ and the structure was optimized with the total energy converged to $10^{-5} \mathrm{eV} /$ supercell. A $3 \times 3 \times 3$ supercell was used in the optimization with a cutoff energy of $480 \mathrm{eV}$ for the plane wave basis sets, a $3 \times 3 \times 3$ Monkhorst-Pack $k$-point mesh, and Fermi smearing with $0.05 \mathrm{eV}$ width. The lattice constant of $\mathrm{LiF}$ after the optimization is $4.07 \AA$ and the error is within $1.3 \%$ compared with experimental results [70].

The ground state energy $E_{\mathrm{tot}}(i, q)$ of defect $i$ with charge $q$ and $E_{\text {tot }}^{\text {bulk }}$ of the perfect $\mathrm{LiF}$ cell were calculated in the VASP-optimized $3 \times 3 \times 3 \mathrm{LiF}$ supercell which is large enough to satisfy the dilute defect condition. To account for interactions from image defects or charges, we studied the scaling behavior of $\Delta E=E_{\mathrm{tot}}(i, q)-E_{\mathrm{tot}}^{\text {bulk }}$ of three representative cases: $\mathrm{Li}_{i}$ (zero charge), $V_{\mathrm{Li}}^{-}$(one negative charge), and $V_{F}^{+}$ (one positive charge). The local structure of the same point defect of $3 \times 3 \times 3 \mathrm{LiF}$ supercell was identical to that in $4 \times 4 \times 4$ supercell calculation and the difference in $\Delta E$ was less than $2 \%$. The charged defects were modeled by adding (for negatively charged defects) or subtracting (for positively charged defects) one background electron to/from the total valence electrons in the supercell. In this study, the electrostatic energy correction $(\Delta V \approx 0.03 \mathrm{eV})$ was obtained from the average electrostatic energy difference between the defected cell (e.g., $V_{\mathrm{Li}}^{-}$) and the perfect cell $[53,71,72]$. We noted that there are other methods to estimate $\Delta V$, e.g., by inspecting the electrostatic potential far away from the

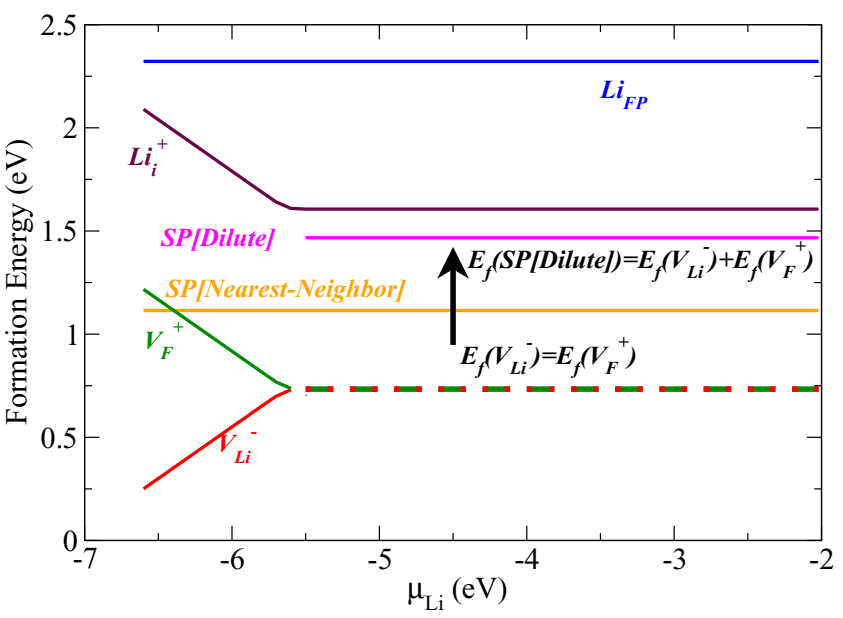

FIG. 1. (Color online) Formation energy $\left(E_{f}\right)$ of several defects from Table I with low formation energies as a function of Li chemical potential $\mu_{\mathrm{Li}}$. The formation energy of each defect was obtained for the self-consistent (zero-charge) Fermi energy at each value of $\mu_{\mathrm{Li}}$.

charged defect [56]. The values of $\Delta V$ obtained from different methods are within the same orders of magnitude $\left(10^{-2} \mathrm{eV}\right)$ and the choice of $\Delta V$ does not change the magnitude of defect concentrations. In addition, a similar magnitude of $\Delta V$ has been observed in the literature [71] for other ionic materials. A neutralization jellium background charge was assumed by VASP to improve the energy convergence with respect to the supercell size [73-75]. The diffusion barriers $E_{m}(i, q)$ of dominant defects were calculated by climbed nudged elastic band method (CI-NEB)[76,77] implemented within VASP.

\section{RESULTS AND DISCUSSION}

\section{A. Formation of defects in $\mathrm{LiF}$}

The formation of defects depends on the properties of the specific material (e.g., band structure), the defects (e.g., defect structure and charge states), and the reservoir (e.g., $\left.\mu_{\mathrm{Li}}\right)$. Figure 1 shows the formation energy $\left(E_{f}\right)$ of several defects with low formation energies as a function of chemical potential $\mu_{\mathrm{Li}}$ of the Li reservoir. Three important constraints are considered in the defect formation in nearly stoichiometric compounds: (1) charge neutrality, which is imposed by Eq. (3), (2) mass conservation, and (3) fixed proportion of cation $\left(\mathrm{Li}^{+}\right)$ and anion $\left(\mathrm{F}^{-}\right)$lattice sites, regardless of their occupancy [6]. In our calculations, the charge neutrality is assumed to be satisfied by bounded charge on defects, free electrons, and free holes. At a high $\mu_{\mathrm{Li}}, V_{\mathrm{Li}}^{-}$and $V_{F}^{+}$are formed with similar formation energies. Since $V_{\mathrm{Li}}^{-}$and $V_{F}^{+}$carry the same amount but opposite charges, the charge neutrality is satisfied by defects in this $\mu_{\mathrm{Li}}$ range. The coexistence of $V_{\mathrm{Li}}^{-}$and $V_{F}^{+}$ can be viewed as the creation of a dilute SP (the black arrow in Fig. 1). The magenta line (marked as $S P$ [Dilute]) denotes the formation energy of a dilute SP when $\mu_{\mathrm{Li}}>-5.5 \mathrm{eV}$. In addition, in this $\mu_{\mathrm{Li}}$ region, it can be noticed that the nearest-neighbor SP (orange solid line in Fig. 1) has a lower formation energy than the dilute SP. However, when $\mu_{\mathrm{Li}}$ is decreased, especially below $-5.5 \mathrm{eV}$, the formation energy of $V_{F}^{+}$is further increased and $V_{\mathrm{Li}}^{-}$formation energy is decreased. 


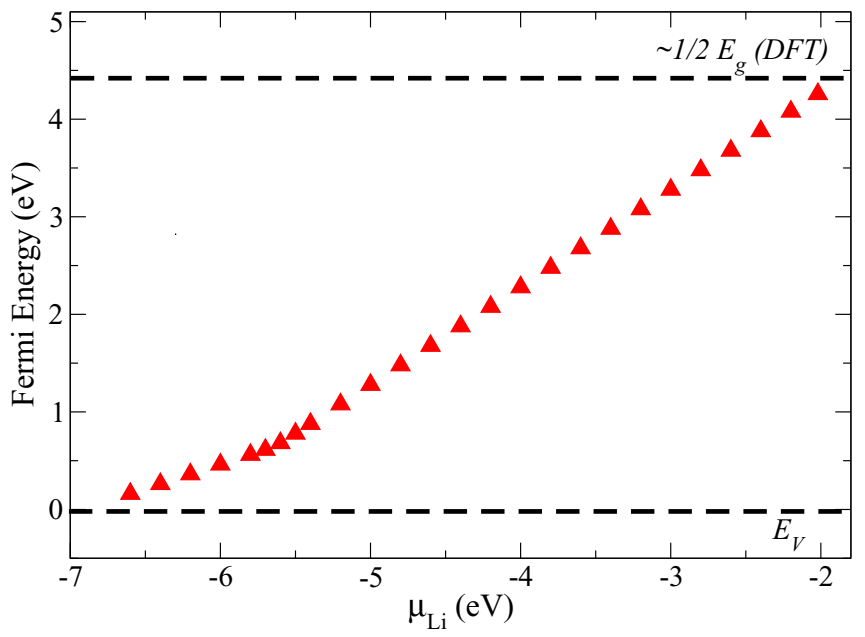

FIG. 2. (Color online) Fermi energy of LiF as a function of the chemical potential $\mu_{\mathrm{Li}}$ in the reservoir.

In this $\mu_{\mathrm{Li}}$ range, the concentrations of $V_{\mathrm{Li}}^{-}$and $V_{F}^{+}$diverge significantly. In order to maintain the charge neutrality, holes are created. In this region, the Fermi level is very close to the top of the valence band (Fig. 2). As a result, holes can be generated by exciting electrons from the valence band to the defect levels $[6,52,53,55]$ induced by $\mathrm{Li}$ vacancies near the Fermi energy. In addition, the mass is conserved between $\mathrm{LiF}$ and $\mathrm{Li} / \mathrm{F}$ reservoirs (constraint 2 ) and the proportion of sites in the calculation system is fixed (constraint 3 ).

In summary, there are two defect formation reactions in $\mathrm{LiF}$ depending on $\mu_{\mathrm{Li}}$ of the Li reservoir as follows.

(1) $\mathrm{LiF}$ on high $\mu_{\mathrm{Li}}$ reservoirs (e.g., Li metal), the main reaction is

$$
\mathrm{Li}_{\mathrm{n}} \mathrm{F}_{\mathrm{n}}-\mathrm{Li}_{\mathrm{R}}-\mathrm{F}_{\mathrm{R}} \stackrel{\Delta G_{i}(\delta r)}{\rightleftharpoons} \mathrm{Li}_{\mathrm{n}-1} \mathrm{~F}_{\mathrm{n}-1}+\mathrm{V}_{\mathrm{Li}}^{-}+\mathrm{V}_{\mathrm{F}}^{+},
$$

where $\mathrm{Li}_{n} \mathrm{~F}_{\mathrm{n}}$ is the perfect $\mathrm{LiF}$ crystal, $\mathrm{Li}_{\mathrm{R}} / \mathrm{F}_{\mathrm{R}}$ refers to the $\mathrm{Li}(\mathrm{F})$ in the reservoir, and this reaction has an increasing activation energy $\Delta G_{i}(\delta r)=E_{f}\left(V_{\mathrm{Li}}^{-}\right)+E_{f}\left(V_{F}^{+}\right)$with increasing the separation $(\delta r)$ of two vacancies.

(2) $\mathrm{LiF}$ on low $\mu_{\mathrm{Li}}$ reservoirs (e.g., $\mathrm{LiCoO}_{2}$ ), the main reaction is

$$
\mathrm{Li}_{\mathrm{n}} \mathrm{F}_{\mathrm{n}}-\mathrm{Li}_{\mathrm{R}} \stackrel{\Delta \mathrm{G}_{\mathrm{p}}}{\rightleftharpoons} \mathrm{Li}_{\mathrm{n}-1} \mathrm{~F}_{\mathrm{n}}+\mathrm{V}_{\mathrm{Li}}^{-}+\oplus
$$

where $\oplus$ is a hole. This reaction has an activation energy $\Delta G_{p}=E_{f}\left(V_{\mathrm{Li}}^{-}\right)$.

According to reactions (15) and (16), we define three defect formation regions depending on $\mu_{\mathrm{Li}}$ of the reservoir (Fig. 3) and the dominant defect concentration as a function of $\mu_{\mathrm{Li}}$ of the reservoir is calculated for each region as follows.

Intrinsic region. In this region, the hole concentration is at least 7-8 orders of magnitude lower than that of Schottky pairs. As a result, the defect formation can be approximated by the reaction (15) and the defect concentration can be estimated by $S\left(V_{\mathrm{Li}}^{-}\right)=S\left(V_{F}^{+}\right)=N_{s} \exp \left(-\Delta G_{i}(\delta r) / k_{B} T\right)$, where, as discussed, $\Delta G_{i}(\delta r)$ depends on the distance of the pair items $V_{\mathrm{Li}}^{-}$and $V_{F}^{+}$. In Fig. 3, the left-triangle line (nearest-neighbor SP with the lowest $\Delta G_{i}$ ) and right-triangle line (dilute SP with the highest $\Delta G_{i}$ ) correspond to the two limits of the concentration of SP in this region, respectively. As a result,

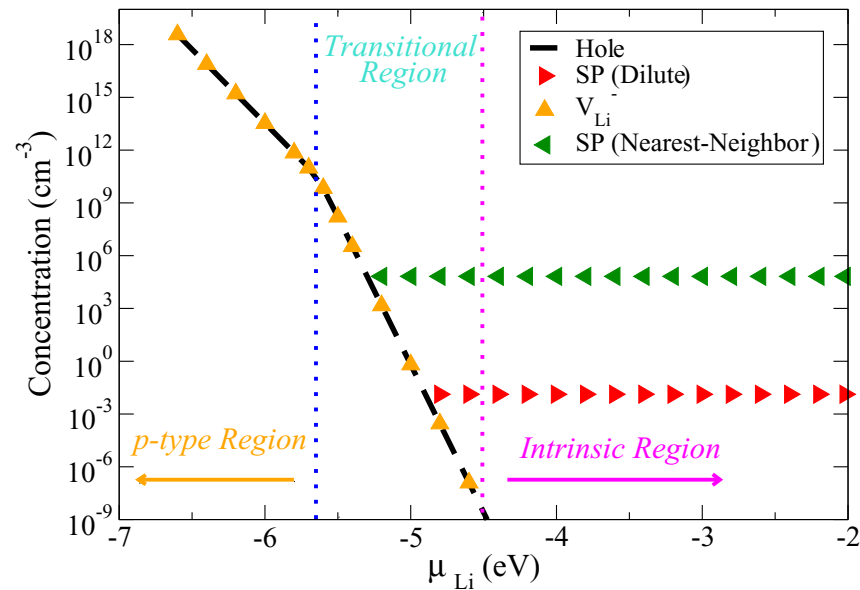

FIG. 3. (Color online) Three regions of defect formation (Kröger-Vink diagram) in $\mathrm{LiF}$ as a function of $\mu_{\mathrm{Li}}$ of the reservoir. $V_{\mathrm{Li}}^{-}$(orange up-triangle) denotes the portion of $V_{\mathrm{Li}}^{-}$whose charge is balanced by hole formation.

the majority ionic carriers in the intrinsic region are $\mathrm{SPs}\left(V_{\mathrm{Li}}^{-}\right.$ and $V_{F}^{+}$pairs).

p-type region. The defect formation is dominated by reaction (16), forming mainly $V_{\mathrm{Li}}^{-}$and the bounded charge is balanced by holes. The corresponding concentration of $V_{\mathrm{Li}}^{-}$ is calculated by $S\left(V_{\mathrm{Li}}^{-}\right)=N_{s} \exp \left(-\Delta G_{p} / k_{B} T\right)$. As a result, the majority ionic carrier in this region is free $V_{\mathrm{Li}}^{-}$.

Transitional region. Defects are formed by both reactions (15) and (16). In this region, the concentrations of $V_{F}^{+}$ and $V_{\mathrm{Li}}^{-}$start to diverge and the dominant defect type changes from SP (intrinsic region) to $V_{\mathrm{Li}}^{-}$( $p$-type region). The excess charge on $V_{\mathrm{Li}}^{-}$which is not balanced by $V_{F}^{+}$is balanced by holes. In order to calculate the defect concentration in this region, we use a charge balance concept by defining a portion $p$ of $V_{\mathrm{Li}}^{-}$whose charge is balanced by $V_{F}^{+}$. By this definition, $1-p$ corresponds to the portion of $V_{\mathrm{Li}}^{-}$, in which the localized negative charge is balanced by holes. The starting point of this region is chosen from the point of $\mu_{\mathrm{Li}}$ that the concentration of holes is about 10 orders of magnitude less than that of dilute SP. This type of $V_{\mathrm{Li}}^{-}$is depicted as up-triangle line in the transitional region depicted in Fig. 3. Therefore, $p$ can be determined by

$$
\frac{1-p}{p}=\frac{n_{\oplus}}{S_{S P}} .
$$

\section{B. Consideration of computational errors and comparison with experiments}

Density functional theory with generalized gradient approximation (DFT-GGA) is known to have its own deficiencies which may lead to errors in the density of states in the conduction band and the value of band gap $\left(E_{g}=E_{C}-E_{V}\right)$ [78]. In our calculations, the influences of several possible errors were considered: (1) the value of $N_{C}$, (2) the value of $\varepsilon_{F}$, and (3) the underestimation of $E_{f}(i, q)$ of defects with the defect induced electronic states occupied (e.g., $V_{\mathrm{Li}}^{-}, \mathrm{Li}_{i}, V_{F}$ ) [79]. To minimize the influence of these errors, we did the following corrections and considerations: (1) $N_{C}$ was corrected by the ratio of the 
effective mass of electrons $\left(m_{\ominus}^{*}\right)$ [69] and holes $\left(m_{\oplus}^{*}\right)$ [80] in LiF with the relationship $N_{C} / N_{V}=\left(m_{\ominus}^{*} / m_{\oplus}^{*}\right)^{3 / 2}[1,2]$, (2) we tested the sensitivity of $\varepsilon_{F}$ to the value of $E_{g}$ by using the experimental value $E_{g}^{\text {expt }}$ [58-60] and found that they were not sensitive to $E_{g}^{\text {DFT-GGA }}$ and $E_{g}^{\text {expt }}\left(E_{g}^{\text {DFT-GGA }}<E_{g}^{\text {expt }}\right)$ in LiF. It is because that $E_{g}^{\text {DFT-GGA }}$ is high enough to prevent free electrons in the conduction band [Eqs. (3) and (6a)] in LiF, and (3) $V_{\mathrm{Li}}^{-}$ is formed by removing a $\mathrm{Li}^{+}$ion from a Li lattice site and the sixfold coordinated $F^{-}$remain as $F^{-}$ions. However, for $V_{\mathrm{Li}}$, one of the nearest-neighbor $F^{-}$ions becomes a neutral $F$ atom. Because the excess electron in the $V_{\mathrm{Li}}^{-}$system stays at the F-2 $p$ orbital (near valence band maximum), DFT-GGA can predict a reliable value of the formation energy of $V_{\mathrm{Li}}^{-}$[79]. In addition, for defects that can have their defect induced electronic states near the conduction band minimum (e.g., $\left.\mathrm{Li}_{i}, V_{F}\right)$, their $S(i, q)$ are very low. We think that the errors that DFT-GGA can overestimate $S(i, q)$ would be insignificant. Therefore, we expect the influence of the errors in determining main defect types negligible.

Our results are consistent with experimental values [8183]. The intrinsic defect formation energy [reaction listed in (15)] was extrapolated from ionic conduction measurement of divalent ion (e.g., $\mathrm{Mg}^{2+}$ ) doped $\mathrm{LiF}$ at elevated temperatures. Traditionally, the formation energy $\Delta G_{\text {exp }}$ was defined according to the mass action law [3,84]

$$
\begin{aligned}
S_{V_{\mathrm{Li}}^{-}} S_{V_{F}^{+}} & =S_{S P}^{2}=N_{s}\left(V_{\mathrm{Li}}^{-}\right) N_{s}\left(V_{F}^{+}\right) e^{-\Delta G_{\text {exp }} / k_{B} T} \\
& =N_{s}\left(V_{\mathrm{Li}}^{-}\right) N_{s}\left(V_{F}^{+}\right) e^{-2 \Delta G_{i} / k_{B} T} .
\end{aligned}
$$

According to the definition of $\Delta G_{\text {exp }}$, our results predict the theoretical two limits for $\Delta G_{\text {exp }}: 2.24 \mathrm{eV}$ (nearest-neighbor $\mathrm{SP}$ ) and $2.94 \mathrm{eV}$ (dilute SP). The experimental value of $\Delta G_{\exp }$ is $\sim 2.68 \mathrm{eV}$ [81-83] with an individual $V_{\mathrm{Li}}^{-}$formation energy $\Delta G_{V_{\mathrm{Li}}^{-}} \sim 0.73 \mathrm{eV}[85]$.

\section{Diffusion barriers of dominant defects}

\section{Diffusion of dilute vacancies in LiF}

We studied the diffusion barriers of the main defects in $\mathrm{LiF}$ $\left(V_{\mathrm{Li}}^{-}\right.$and $\left.V_{F}^{+}\right)$through CI-NEB calculations [76,77]. Since $V_{\mathrm{Li}}^{-}$ and $V_{F}^{+}$sites maintain the same symmetry, we constructed two diffusion pathways for each: through face center (FC, along [110] direction) and through body center (BC, along [11 1 ] direction). For $V_{\mathrm{Li}}^{-}$, after relaxation, the saddle point of the constructed $\mathrm{BC}$ path relaxed to a point between $\mathrm{FC}$ and BC (shown in Fig. 4 inset: path 2). The diffusion barrier for $V_{\mathrm{Li}}^{-}$is slightly smaller for path $2(\sim 0.57 \mathrm{eV})$ than that for path 1 (FC path, $\sim 0.60 \mathrm{eV}$ ), although path 2 has a larger travel distance than path 1 . However, for $V_{F}^{+}$, two constructed pathways relaxed to the same saddle point (FC path) with a $0.69 \mathrm{eV}$ diffusion barrier. The higher diffusion barrier for $V_{F}^{+}$ can be attributed to that $F^{-}$has a larger ionic radii compared with $\mathrm{Li}^{+}$.

\section{Diffusion of nearest-neighbor Schottky pair}

It is important to discuss the motion of neutral nearestneighbor SP and its contribution to the ionic conductivity, since it has a higher concentration compared with dilute SP in the intrinsic region. We considered two mechanisms for

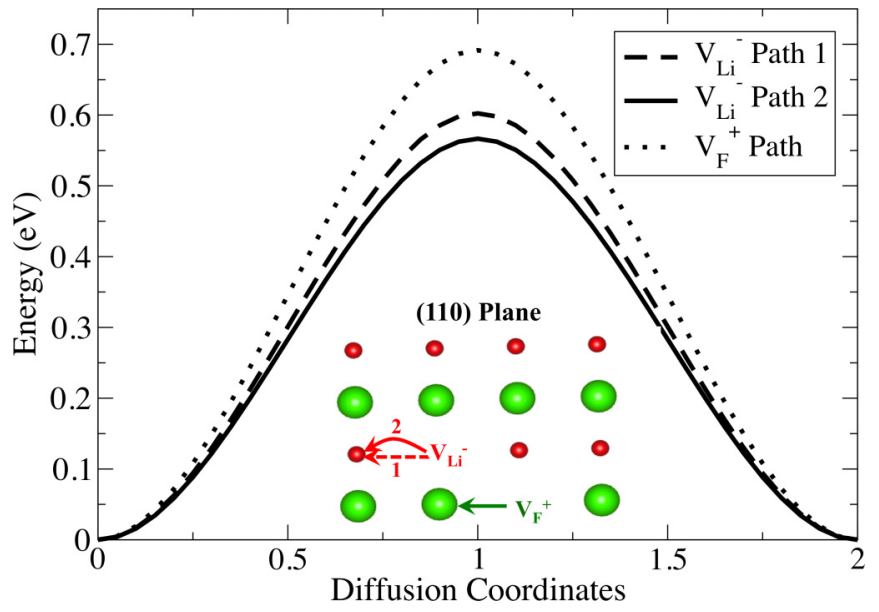

FIG. 4. (Color online) Diffusion barriers of $V_{\mathrm{Li}}^{-}$through paths 1 , 2 and $V_{F}^{+}$through the face center path. Inset: schematic figure shows diffusion pathways for $V_{\mathrm{Li}}^{-}$and $V_{F}^{+}$.

nearest-neighbor SP to diffuse in the LiF: (1) nearest-neighbor SP diffuses together to its nearest available site and (2) nearestneighbor SP separates to $V_{\mathrm{Li}}^{-}$and $V_{F}^{+}$and then diffuse. In an ionic conductivity measurement [84], the first mechanism is not counted since the nearest-neighbor SP does not carry any net charge [Eq. (13)].

As a result, the second mechanism is considered in our ionic conductivity calculations. The disassociation energy barrier ( $\Delta G_{d}$, shown in Fig. 5) is estimated based on two assumptions: (1) $V_{\mathrm{Li}}^{-}$is more mobile than $V_{F}^{+}$(Sec. IIIC 1) and (2) nearest-neighbor SP dissociates when $V_{\mathrm{Li}}^{-}$diffuses to its next nearest neighbor (shown in inset of Fig. 5). This disassociation energy $(\approx 0.6 \mathrm{eV})$ is added to estimate the contribution of nearest-neighbor SP to ionic conduction. The contribution of nearest-neighbor SP to the total ionic conductivity is about five orders of magnitude lower than that from dilute SP at room temperature. Although the second nearest-neighbor SP is not well separated (about $0.1 \mathrm{eV}$ lower in energy than dilute SP), we would expect the contribution of nearest-neighbor SP to be very small.

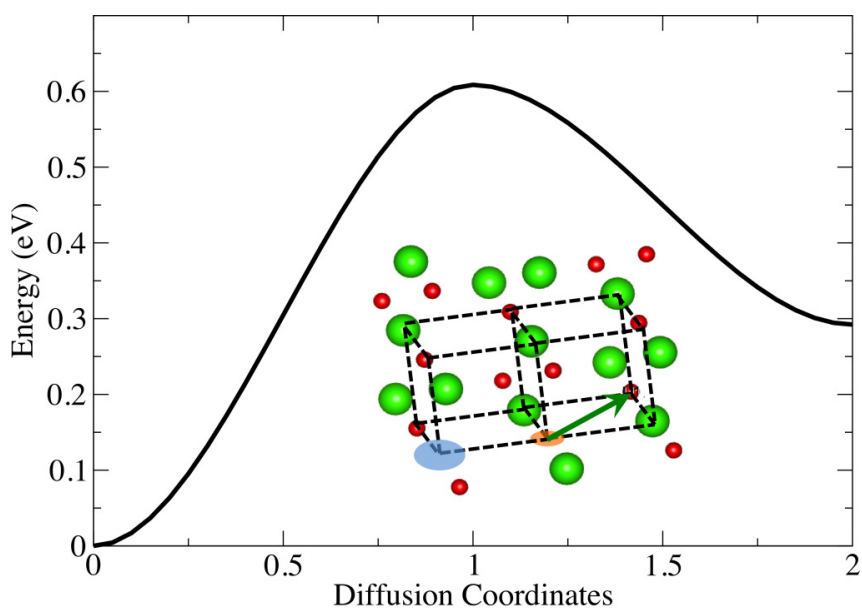

FIG. 5. (Color online) Disassociation energy of a nearestneighbor Schottky pair. Inset: schematic figure shows the calculated pathway for the nearest-neighbor Schottky pair disassociation. 


\section{Ionic conduction in $\mathrm{LiF}$}

The ionic conductivity is calculated according to the main defects in each region defined in Sec. III A.

Intrinsic region:

$$
\begin{aligned}
\sigma & =\sigma_{S P_{d}}+\sigma_{S P_{n}} \\
& \approx \frac{q^{2} F^{2}}{R T}\left[S_{S P_{d}}+S_{S P_{n}} \exp \left(\frac{-\Delta G_{d}}{k_{B} T}\right)\right]\left(D_{V_{\mathrm{Li}}^{-}}+D_{V_{F}^{+}}\right),
\end{aligned}
$$

where $\Delta G_{d}$ is the disassociation energy calculated in Sec. III C $2 ; S_{S P_{d}}$ and $S_{S P_{n}}$ are the concentration of dilute and nearest-neighbor SP. Since $V_{F}^{+}$has $\sim 0.12 \mathrm{eV}$ higher in migration barrier compared with $V_{\mathrm{Li}}^{-}$, its contribution is insignificant to the total ionic conduction.

Transitional region:

$$
\begin{aligned}
\sigma & =\sigma_{V_{\mathrm{Li}}^{-}}+\sigma_{S P_{d}}+\sigma_{S P_{n}} \approx \sigma_{V_{\mathrm{Li}}^{-}}+\sigma_{S P_{d}} \\
& \approx \frac{q^{2} F^{2}}{R T}\left[p S_{V_{\mathrm{Li}}^{-}}\left(D_{V_{\mathrm{Li}}^{-}}+D_{V_{F}^{+}}\right)+(1-p) S_{V_{\mathrm{Li}}^{-}} D_{V_{\mathrm{Li}}^{-}}\right],
\end{aligned}
$$

where $p$ is the portion of $V_{\mathrm{Li}}^{-}$whose charge is balanced by the formation of $V_{F}^{+}$as defined in Sec. III A. In this region, we neglect the contribution from nearest-neighbor SP due to the following: (1) its small contribution compared with dilute SP; (2) in this region, the contribution from $V_{\mathrm{Li}}^{-}$becomes dominant especially when approaching $p$-type region.

p-type region:

$$
\sigma=\sigma_{V_{\mathrm{Li}}^{-}}=\frac{F^{2} q^{2}}{R T} S_{V_{\mathrm{Li}}^{-}} D_{V_{\mathrm{Li}}^{-}} .
$$

The calculated results are shown in Fig. 6. The activation energy $\left(E_{f}+E_{m} \approx 2.03 \mathrm{eV}\right)$ in the intrinsic region is comparable with experimental extrapolation $(1.99 \mathrm{eV})$ [83] to the intrinsic region at room temperature. The ionic conductivity is small in the intrinsic region (high $\mu_{\mathrm{Li}}$ ) and is increased dramatically when $\mu_{\mathrm{Li}}$ is below $-4.6 \mathrm{eV}$. This dramatic increase in ionic conductivity is due to the formation of $V_{\mathrm{Li}}^{-}$ which is preferred at low $\mu_{\mathrm{Li}}$ and it is no longer constrained by the formation of $V_{F}^{+}$to balance the charge (Fig. 3).

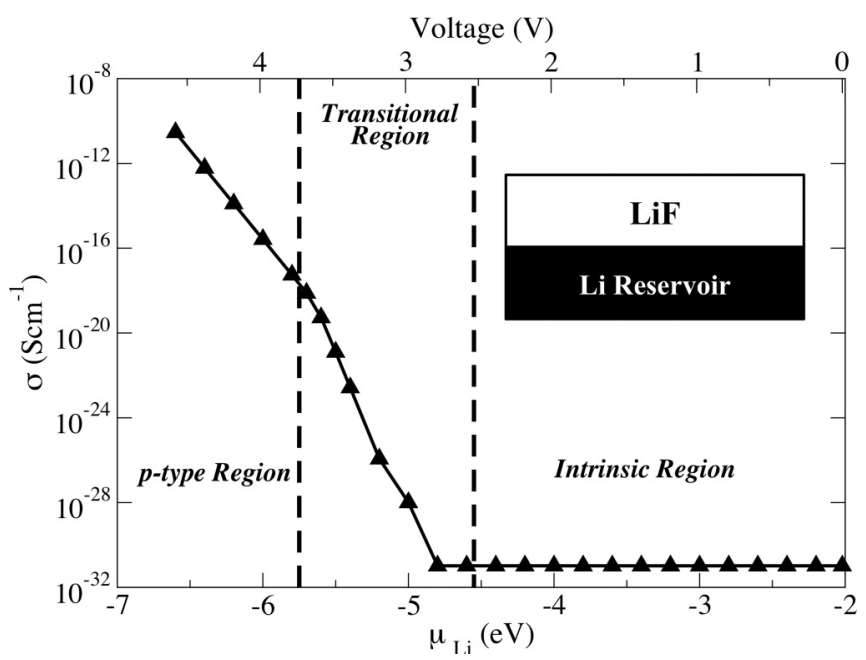

FIG. 6. Ionic conductivity of $\mathrm{LiF}$ as a function of the chemical potential of Li reservoir. Inset: schematic drawing of $\mathrm{LiF}$ contacting a Li reservoir (e.g., an electrode for application of LIBs).

\section{E. LiF as an engineered SEI for LIBs}

Considering a solid electrolyte (an engineered artificial $\mathrm{SEI}$ ) coated on electrodes for LIBs, the $\mathrm{Li}$ reservoir (inset in Fig. 6) can be viewed as an electrode on which the $\mathrm{LiF}$ is coated. For different electrodes (e.g., $\mu_{\mathrm{Li}}^{\mathrm{Li}-m e t a l} \sim-2.02$ $\left.\mathrm{eV} ; \mu_{\mathrm{Li}}^{\mathrm{LiCO}_{2}}=-6.2 \mathrm{eV}\right)$ or electrodes with different state of charge (e.g., $\mathrm{LiC}_{12}, \mathrm{LiC}_{6}$ ), the values of $\mu_{\mathrm{Li}}$ are different. In electrochemistry, the electrochemical potential of $\mathrm{Li}^{+}$in a spatial point is defined as [86]

$$
\tilde{\mu}_{\mathrm{Li}^{+}}=\mu_{\mathrm{Li}^{+}}+z_{i} F \phi,
$$

where $\tilde{\mu}_{\mathrm{Li}^{+}}$is the electrochemical potential, $\mu_{\mathrm{Li}^{+}}$is the chemical potential of $\mathrm{Li}^{+}, z_{i}\left(z_{i}=1\right.$ for $\left.\mathrm{Li}^{+}\right)$is the associated charge, and $\phi$ is the electrical potential in that spatial point. At thermodynamic equilibrium, the electrochemical potential is the same everywhere in a battery (i.e., $\tilde{\mu}_{\mathrm{Li}^{+}}^{\text {cadhode }}=\tilde{\mu}_{\mathrm{Li}^{+}}^{\text {anode }}$ ). Under this condition, we can estimate the cell voltage by

$$
V=\phi^{\text {cathode }}-\phi^{\text {anode }}=-\frac{\mu_{\mathrm{Li}^{+}}^{\text {cathode }}-\mu_{\mathrm{Li}^{+}}^{\text {anode }}}{F} .
$$

By assuming $\mu_{\mathrm{Li}} \approx \mu_{\mathrm{Li}^{+}}+\mu_{\ominus}$ in the electrode and the same wire (e.g., copper) used to connect the cathode and anode $\left(\mu_{\ominus}^{\text {cathode }}=\mu_{\ominus}^{\text {anode }}\right)$, the voltage of the cell can be estimated from the differences of $\mu_{\mathrm{Li}}$ in electrodes. This is the wellknown Nernst equation in electrochemistry [7]. In LIBs, $\mu_{\mathrm{Li}}$ in Li metal is typically defined as a reference (zero volt). Therefore, we can relate $\mu_{\mathrm{Li}}$ in the reservoir to the open-circuit voltage of the electrodes (a half cell) by referencing to $\mu_{\mathrm{Li}}$ in Li metal

$$
V=-\left(\mu_{\mathrm{Li}}-\mu_{\mathrm{Li}}^{\mathrm{metal}}\right) / e .
$$

The ionic conductivity $\sigma$ in LiF highly depends on the electrode it contacts with. For example, if $\mathrm{LiF}$ is coated on a negative electrode surface (e.g., graphite, silicon), $\sigma$ is very small $\left(\approx 10^{-30} \mathrm{~S} \mathrm{~cm}^{-1}\right)$. Compared with $\sigma$ in other SEI species (e.g., $\mathrm{Li}_{2} \mathrm{CO}_{3}[20,21]$ ), $\sigma$ in $\mathrm{LiF}$ is 18 to 20 orders of magnitude lower. However, if it is coated on a positive electrode (e.g., $\left.\mathrm{LiCoO}_{2}\right), \sigma$ is controlled by free $V_{\mathrm{Li}}^{-}$diffusion and it is comparable with other SEI species (e.g., $\mathrm{Li}_{2} \mathrm{CO}_{3}$ [20,21]). This result is significant since it demonstrates that the ionic conductivity of LiF is strongly voltage dependent. Therefore, higher doping of divalent cation (e.g., $\mathrm{Mg}^{2+}$ ) is suggested to improve $\mathrm{Li}$ ion conductivity of $\mathrm{LiF}$ coating on negative electrodes by increasing the concentration of diffusion carrier $V_{\mathrm{Li}}^{-}$.

The ionic transport contributes to the electrical conduction in ionic crystals (e.g., LiF) [1,2]. It can be expected that $\mathrm{LiF}$ is more electrical insulating on the negative electrode than other species (e.g., $\mathrm{Li}_{2} \mathrm{CO}_{3}[20,21]$ ) due to its low ionic conductivity. It was believed that the electron leakage from the electrode is an important step in the ethylene carbonate decomposition leading to SEI formation [22-24]. Various groups have reported fluoroethylene carbonate (FEC) as an electrolyte additive that can improve the performance of $\mathrm{Si}$ electrode with increased $\mathrm{LiF}$ formation in the SEI layer [42-47]. Our results provide a reason for this observation that $\mathrm{LiF}$ on the negative electrode surface can better passivate the electrode surface and prevent electron leakage. As a result, 
it can cause less capacity loss and longer cycling life of the electrode.

\section{CONCLUSIONS}

We developed a method to study the defect physics in a material in equilibrium with an external phase. This method establishes a correlation between the open-circuit voltage and Li chemical potential in the electrodes. We applied this approach to study defect reactions in $\mathrm{LiF}$ in contact with different electrode materials which serve as Li reservoirs. We demonstrated that the defect formation in a solid electrolyte can be affected by the open-circuit voltage of the electrode, which is a measurable and controllable parameter. Three regions (intrinsic, transitional, and $p$-type) were defined to describe the main defect reactions with different electrodes. We found that the main defect type in the intrinsic region (high $\mu_{\mathrm{Li}}$ reservoirs) was Schottky pair and in the $p$-type region (low $\mu_{\mathrm{Li}}$ reservoirs) was Li-ion vacancy. The diffusion barrier of main defects in each region was estimated from CI-NEB calculation and mapped to ionic conduction based on the Nernst-Einstein formula. The ionic conductivity is very low $\left(10^{-31} \mathrm{~S} \mathrm{~cm}^{-1}\right)$ in the intrinsic region and increases (to $10^{-12} \mathrm{~S} \mathrm{~cm}^{-1}$ when $\mu_{\mathrm{Li}} \approx-6.5 \mathrm{eV}$ ) with increasing the voltage of the nearby electrode. Thus, the ionic conduction in a solid electrolyte is very sensitive to the external potential of the electrode on which the electrolyte material is coated. This work extends the well known relationship between defect concentration and chemical potential for gas phase reactions to electrochemical reactions.

\section{ACKNOWLEDGMENTS}

J.P., Y.T.C., and Y.Q. gratefully acknowledge the support by Department of Energy and the Assistant Secretary for Energy Efficiency and Renewable Energy (Office of Vehicle Technologies of the U.S. Department of Energy under Contract No. DE-AC02-05CH11231, Subcontract No. 7056410) under the Advanced Battery Materials Research (BMR) Program. J.P. and Y.T.C. acknowledge the support from National Science Foundation Grant No. 1355438 (Powering the Kentucky Bioeconomy for a Sustainable Future) and the Center for Computational Sciences at University of Kentucky. J.P., Y.T.C., and Y.Q. would like to sincerely thank the referees for their kind suggestions during the peer-review process.
[1] C. Kittel, Introduction to Solid State Physics (Wiley, New York, 2005).

[2] N. Ashcroft and N. Mermin, Solid State Physics, Science: Physics (Saunders College, Philadelphia, 1976).

[3] J. Maier, Physical Chemistry of Ionic Materials: Ions and Electrons in Solids (Wiley, New York, 2004).

[4] K. Takada, Acta Mater. 61, 759 (2013).

[5] F. A. Kröger and H. J. Vink, Solid state physics 3, 307 (1956).

[6] R. Swalin, Thermodynamics of Solids (Wiley, New York, 1972).

[7] R. Huggins, Advanced Batteries: Materials Science Aspects, SpringerLink: Springer e-Books (Springer, New York, 2008).

[8] Z. Liu, W. Fu, E. A. Payzant, X. Yu, Z. Wu, N. J. Dudney, J. Kiggans, K. Hong, A. J. Rondinone, and C. Liang, J. Am. Chem. Soc. 135, 975 (2013).

[9] N. Kamaya, K. Homma, Y. Yamakawa, M. Hirayama, R. Kanno, M. Yonemura, T. Kamiyama, Y. Kato, S. Hama, K. Kawamoto, and A. Mitsui, Nat. Mater. 10, 682 (2011).

[10] N. D. Lepley, N. A. W. Holzwarth, and Y. A. Du, Phys. Rev. B 88, 104103 (2013).

[11] Y. Mo, S. P. Ong, and G. Ceder, Chem. Mater. 24, 15 (2012).

[12] Y. A. Du and N. A. W. Holzwarth, Phys. Rev. B 76, 174302 (2007).

[13] Y. A. Du and N. A. W. Holzwarth, Phys. Rev. B 81, 184106 (2010).

[14] Y. A. Du and N. A. W. Holzwarth, Phys. Rev. B 78, 174301 (2008).

[15] Y. J. A. Du and N. A. W. Holzwarth, J. Electrochem. Soc. 154, A999 (2007).

[16] J. Li, C. Ma, M. Chi, C. Liang, and N. J. Dudney, Adv. Energy Mater. 5 (2015).

[17] D. Aurbach, J. Power Sources 89, 206 (2000).

[18] P. Verma, P. Maire, and P. Novak, Electrochim. Acta 55, 6332 (2010).
[19] S. Q. Hao and C. Wolverton, J. Phys. Chem. C 117, 8009 (2013).

[20] S. Q. Shi, P. Lu, Z. Y. Liu, Y. Qi, L. G. Hector, H. Li, and S. J. Harris, J. Am. Chem. Soc. 134, 15476 (2012).

[21] S. Q. Shi, Y. Qi, H. Li, and L. G. Hector, J. Phys. Chem. C 117, 8579 (2013).

[22] K. Leung and J. L. Budzien, Phys. Chem. Chem. Phys. 12, 6583 (2010).

[23] K. Leung, J. Phys. Chem. C 117, 1539 (2013).

[24] K. Leung, Y. Qi, K. R. Zavadil, Y. S. Jung, A. C. Dillon, A. S. Cavanagh, S. H. Lee, and S. M. George, J. Am. Chem. Soc. 133, 14741 (2011).

[25] Q. L. Zhang, X. C. Xiao, Y. T. Cheng, and M. W. Verbrugge, Appl. Phys. Lett. 105, 061901 (2014).

[26] H. Wu, G. Chan, J. W. Choi, I. Ryu, Y. Yao, M. T. McDowell, S. W. Lee, A. Jackson, Y. Yang, L. B. Hu, and Y. Cui, Nat. Nanotechnol. 7, 310 (2012).

[27] M. N. Obrovac, L. Christensen, D. B. Le, and J. R. Dahnb, J. Electrochem. Soc. 154, A849 (2007).

[28] L. Y. Beaulieu, K. W. Eberman, R. L. Turner, L. J. Krause, and J. R. Dahn, Electrochem. Solid State 4, A137 (2001).

[29] T. D. Hatchard and J. R. Dahn, J. Electrochem. Soc. 151, A838 (2004).

[30] T. Song, J. L. Xia, J. H. Lee, D. H. Lee, M. S. Kwon, J. M. Choi, J. Wu, S. K. Doo, H. Chang, W. Il Park, D. S. Zang, H. Kim, Y. G. Huang, K. C. Hwang, J. A. Rogers, and U. Paik, Nano Lett. 10, 1710 (2010).

[31] L. Y. Beaulieu, T. D. Hatchard, A. Bonakdarpour, M. D. Fleischauer, and J. R. Dahn, J. Electrochem. Soc. 150, A1457 (2003).

[32] X. C. Xiao, P. Lu, and D. Ahn, Adv. Mater. 23, 3911 (2011).

[33] Y. S. Jung, A. S. Cavanagh, L. A. Riley, S. H. Kang, A. C. Dillon, M. D. Groner, S. M. George, and S. H. Lee, Adv. Mater. 22, 2172 (2010). 
[34] Z. Chen, Y. Qin, K. Amine, and Y.-K. Sun, J. Mater. Chem. 20, 7606 (2010).

[35] D. W. Liu and G. Z. Cao, Energ. Environ. Sci. 3, 1218 (2010).

[36] S. S. Zhang, J. Power Sources 162, 1379 (2006).

[37] K. Yang, L. Z. Fan, J. Guo, and X. H. Qu, Electrochim. Acta 63, 363 (2012).

[38] Y. Q. Wang, L. Guo, Y. G. Guo, H. Li, X. Q. He, S. Tsukimoto, Y. Ikuhara, and L. J. Wan, J. Am. Chem. Soc. 134, 7874 (2012).

[39] L. B. Chen, K. Wang, X. H. Xie, and J. Y. Xie, Electrochem. Solid State 9, A512 (2006).

[40] L. B. Chen, K. Wang, X. H. Xie, and J. Y. Xie, J. Power Sources 174, 538 (2007).

[41] M. Nie, D. Chalasani, D. P. Abraham, Y. Chen, A. Bose, and B. L. Lucht, J. Phys. Chem. C 117, 1257 (2013).

[42] R. Elazari, G. Salitra, G. Gershinsky, A. Garsuch, A. Panchenko, and D. Aurbach, J. Electrochem. Soc. 159, A1440 (2012).

[43] V. Etacheri, O. Haik, Y. Goffer, G. A. Roberts, I. C. Stefan, R. Fasching, and D. Aurbach, Langmuir 28, 965 (2012).

[44] K. Fridman, R. Sharabi, R. Elazari, G. Gershinsky, E. Markevich, G. Salitra, D. Aurbach, A. Garsuch, and J. Lampert, Electrochem. Commun. 33, 31 (2013).

[45] E. Markevich, K. Fridman, R. Sharabi, R. Elazari, G. Salitra, H. E. Gottlieb, G. Gershinsky, A. Garsuch, G. Semrau, M. A. Schmidt, and D. Aurbach, J. Electrochem. Soc. 160, A1824 (2013).

[46] M. Y. Nie, D. P. Abraham, Y. J. Chen, A. Bose, and B. L. Lucht, J. Phys. Chem. C 117, 13403 (2013).

[47] I. A. Profatilova, C. Stock, A. Schmitz, S. Passerini, and M. Winter, J. Power Sources 222, 140 (2013).

[48] G. A. Baraff and M. Schlüter, Phys. Rev. Lett. 55, 1327 (1985).

[49] W. Shockley and J. L. Moll, Phys. Rev. 119, 1480 (1960).

[50] W. Walukiewicz, Appl. Phys. Lett. 54, 2094 (1989).

[51] B. P. R. Marioton and U. Gösele, J. Appl. Phys. 63, 4661 (1988).

[52] S. B. Zhang and J. E. Northrup, Phys. Rev. Lett. 67, 2339 (1991).

[53] D. B. Laks, C. G. Van de Walle, G. F. Neumark, P. E. Blöchl, and S. T. Pantelides, Phys. Rev. B 45, 10965 (1992).

[54] J.-L. Zhao, W. Zhang, X.-M. Li, J.-W. Feng, and X. Shi, J. Phys.: Condens. Matter 18, 1495 (2006).

[55] J. Neugebauer and C. G. Van de Walle, Phys. Rev. B 50, 8067 (1994).

[56] C. G. Van de Walle and J. Neugebauer, J. Appl. Phys. 95, 3851 (2004).

[57] Q. Zhang, X. Xiao, W. Zhou, Y.-T. Cheng, and M. W. Verbrugge Adv. Energy Mater. 5 (2015).
[58] D. M. Roessler and W. C. Walker, J. Phys. Chem. Solids 28, 1507 (1967).

[59] R. C. Chaney, E. E. Lafon, and C. C. Lin, Phys. Rev. B 4, 2734 (1971).

[60] M. Piacentini, D. W. Lynch, and C. G. Olson, Phys. Rev. B 13, 5530 (1976).

[61] J. B. Goodenough and Y. Kim, Chem. Mater. 22, 587 (2010).

[62] A. Van der Ven, G. Ceder, M. Asta, and P. D. Tepesch, Phys. Rev. B 64, 184307 (2001).

[63] G. Dolling, H. Smith, R. Nicklow, P. Vijayaraghavan, and M. Wilkinson, Phys. Rev. 168, 970 (1968).

[64] C. A. Sholl, J. Phys. C 14, 2723 (1981).

[65] G. Kresse and J. Furthmüller, Phys. Rev. B 54, 11169 (1996).

[66] G. Kresse and J. Furthmuller, Comput. Mater. Sci. 6, 15 (1996).

[67] J. P. Perdew, K. Burke, and M. Ernzerhof, Phys. Rev. Lett. 77, 3865 (1996).

[68] G. Kresse and D. Joubert, Phys. Rev. B 59, 1758 (1999).

[69] D. Sirdeshmukh, L. Sirdeshmukh, and K. Subhadra, Alkali Halides: A Handbook of Physical Properties, Materials Science Series (Springer, New York, 2001).

[70] C. A. Hutchison and H. L. Johnston, J. Am. Chem. Soc. 62, 3165 (1940).

[71] R. M. Jacobs, J. H. Booske, and D. Morgan, Phys. Rev. B 86, 054106 (2012).

[72] S. Lany and A. Zunger, Modell. Simul. Mater. Sci. Eng. 17, 084002 (2009).

[73] G. Makov and M. C. Payne, Phys. Rev. B 51, 4014 (1995).

[74] J. Neugebauer and M. Scheffler, Phys. Rev. B 46, 16067 (1992).

[75] M. Leslie and M. J. Gillan, J. Phys. C: Solid State 18, 973 (1985).

[76] G. Henkelman and H. Jonsson, J. Chem. Phys. 113, 9978 (2000).

[77] G. Henkelman, B. P. Uberuaga, and H. Jonsson, J. Chem. Phys. 113, 9901 (2000).

[78] R. W. Godby, M. Schlüter, and L. J. Sham, Phys. Rev. Lett. 56, 2415 (1986).

[79] P. Rinke, A. Janotti, M. Scheffler, and C. G. Van de Walle, Phys. Rev. Lett. 102, 026402 (2009).

[80] R. M. Nieminen, J. Phys. C: Solid State 8, 2077 (1975).

[81] Y. Haven, Recl. Trav. Chim. Pay.-B. 69, 1471 (1950).

[82] T. G. Stoebe and R. A. Huggins, J. Mater. Sci. 1, 117 (1966).

[83] S. Pizzini, J. Appl. Electrochem. 1, 153 (1971).

[84] R. W. Dreyfus and A. S. Nowick, Phys. Rev. 126, 1367 (1962).

[85] O. S. Spencer, J. Appl. Phys. 40, 168 (1969).

[86] A. McNaught and A. Wilkinson, Compendium of Chemical Terminology: IUPAC Recommendations, IUPAC Chemical Data Series (Blackwell Science, London, 1997). 